\title{
VOLUME OF FLIUDS INTERFACE RECONSTRUCTION WITH CURVATURE AND CORNER DEFINITIONS
}

\author{
JIN YAO'， JERRY LIU ${ }^{1,2}$ \\ ${ }^{1}$ Lawrence Livermore National Laboratory \\ 7000 East Avenue, Livermore, CA 94551, USA \\ yao2@1lnl.gov, https://www.linkedin.com/in/jin-yao-1936a370/ \\ ${ }^{2}$ Duke University \\ 1030 Hollyburne Ave., Durham, NC 27708, USA \\ Liu79@1lnl.gov, jerry.w.liu@duke.edu, https://github.com/jerrywliu
}

Key words: High Order, Interface Reconstruction, Curvature, Corner, Non-Diffusive Advection.

\begin{abstract}
Interface reconstruction with VOF (volume of fluids) is an essential phase in an ALE (Arbitrary Lagrange \& Eulerian) simulation. Some historical issues associated with the Youngs method have not been well addressed. One is that the slope estimate is not always accurate. A more serious issue is that the interface is discontinuous on cell faces with a curved interface geometry. Plus, with an existing VOF method a corner cannot be reconstructed in general.
\end{abstract}

We propose a new VOF approach in order to address these issues. We treat the case of a single material and void, and the partial volumes in mixed cells exactly given. We assume the nodes not owned by any pure elements are sparsely distributed in a mesh. This is generally true for a moderate curvature. Then, most mixed cell nodes can be coloured with pure elements, and can provide orientation of interface facets. For a given interior mixed zone in two-dimensions, we find its mixed neighbours and three partial volumes are given. A linear facet has two degrees of freedom therefore can be reconstructed with a local optimization with volume matching. A quadratic facet (with three degrees of freedom) can be computed exactly that matches the known partial volumes. This is to say a planar interface geometry can be locally exactly reconstructed away from a corner, and interface curvature can be calculated with neighbour volume fractions. The case of a corner can be identified with a sudden slope change and high curvature with noticeable gaps between neighbour facets. Then, a local optimization for matching volumes can again be performed and the corner is reconstructed.

We have implemented this algorithm in 2D at the Lawrence Livermore National Laboratory. Preliminary tests show that exact planar geometries can be reconstructed, and corners can be accurately defined. In the case of a curved geometry, the gaps between neighbour facets are of high order. When necessary, the gaps between facets can be eliminated by a local modification of a facet without affecting the order of accuracy. The proposed algorithm is by nature applicable to an arbitrarily given mesh because the only necessary requirement is a function to accurately compute the partial volume bounded by a facet (linear, corner, circular, or conic). A similar approach is expected to work in 3D provided an accurate method to compute volumes. 


\section{INTRODUCTION}

Youngs method has been successful over decades for computing the partial volume distributions into relaxed mesh and helping the remapping phase. Because an estimate of interface slope is utilized, a second order accuracy is achieved with Youngs method if the slope is obtained properly. This order of accuracy is sufficient for advection in most situations. However, because slope is the only determining factor added to the volume fraction to reconstruct an interface in a mixed cell, the geometry of a facet is a linear one. In the case of a curved interface, the linear interface facets are by nature disjointed. Likewise, a given slope is not sufficient either to handle the case that a corner is present in a mixed cell.

To address the above issues with Youngs method, we recently implemented a new volume of fluids (VOF) interface reconstruction method at Lawrence Livermore National Laboratory. We choose curved interface facet for reconstruction and our results indicate the gaps between neighbor facets can be eliminated and corners can be reconstructed as well. A major difference between the new method and Youngs method is, instead of trying to compute a facet slope, we match three neighbor volumes exactly using a Newton method for a circular fit of interface facet (a linear segment is considered a degenerated case of an arc). In this way the local solution is final and consistent with a planar boundary consisting of straight or circular sides. An immediate benefit is that such a local solution can be extrapolated to predict a neighboring facet that has an unknown orientation. A volume matching is then applied to correct the neighbor fact and obtain its final solution.

Reconstructing a too difficult because

The proposed method is of $3^{\text {td }}$ order accuracy in space.
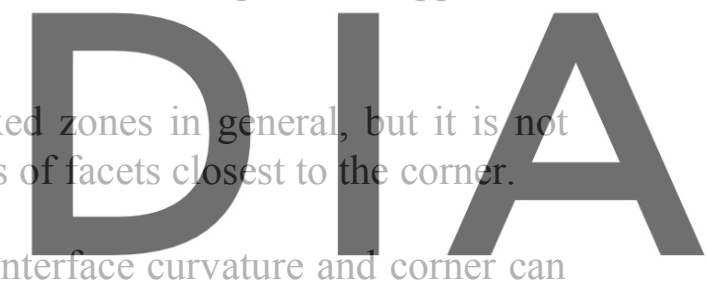

be computed correctly. We will demonstrate our reconstruction scheme with basic planar

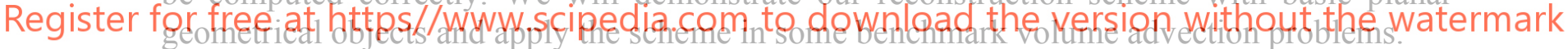

\section{THE PROPOSED RECONSTRUCTION SCHEME}

To start with we assume the input mesh is locally fine enough such that the curvature of interface contained in any mixed zone that does not contain a corner is moderate (with radius of curvature greater than 2 times the local zone size, say). A proper mesh resolution would allow cases of interface double crossing a zone-edge and cases of corners to be isolated.

\subsection{Orientation of an interface facet}

A facet is the solution of interface reconstruction contained in a mixed zone. An essential issue with an interface reconstruction method is how to determine the orientation of a facet (assumed moderately curved or straight, if not a corner). In this work we deal with the case of only two materials, with ids "-1" and "1" (or "-_" and "+") for "below" and "above" interface and utilize the fact that almost all nodes owned by mixed zones are also owned by pure zones if resolution is fine enough. To be specific we assume a given resolution allows only one 
piece of interface contained in any mixed zone. Then, we can "color" a node of a mixed zone with the material ID of a pure zone that also owns this node. A node owned by both a void zone and a full zone is colored " 0 " and is right on interface. The orientation of a facet in a mixed zone can be determined if all nodes are colored as demonstrated in the figure below.
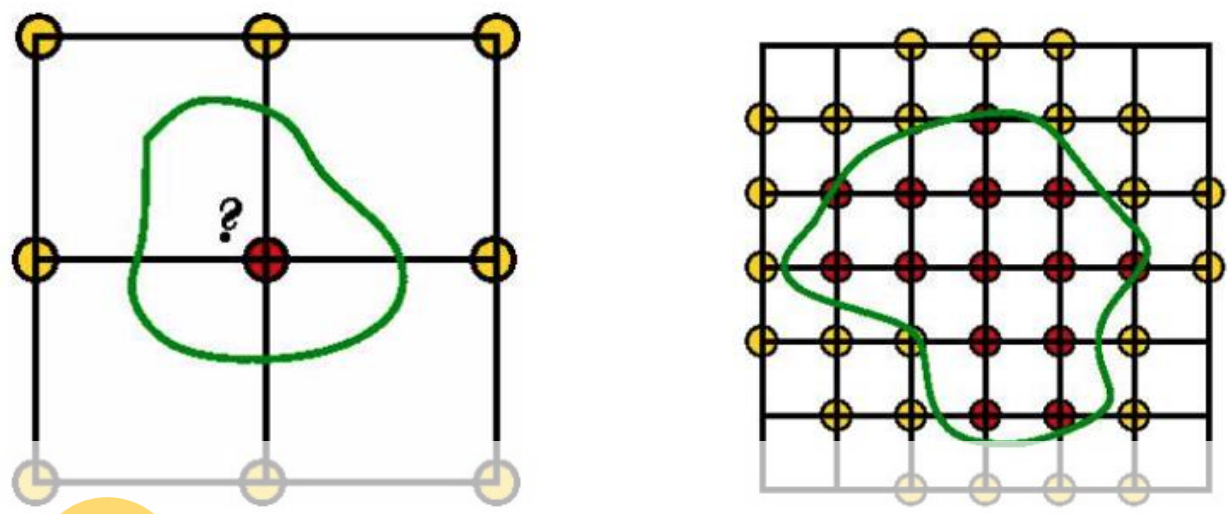

Figure 1: Left: an insufficient resolution; right: a good resolution. Red/yellow nodes are on the full/void sides.

\subsection{Neighbor finding for regular zones}

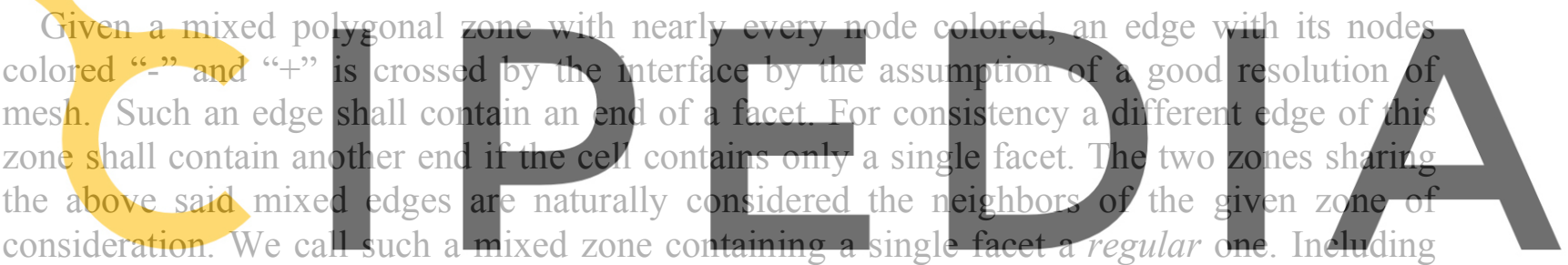

itself, there are three local mixed neighbors associated with an interior regular mixed zone.

Register for free at https//www.scipedia.com to download the version without the watermark
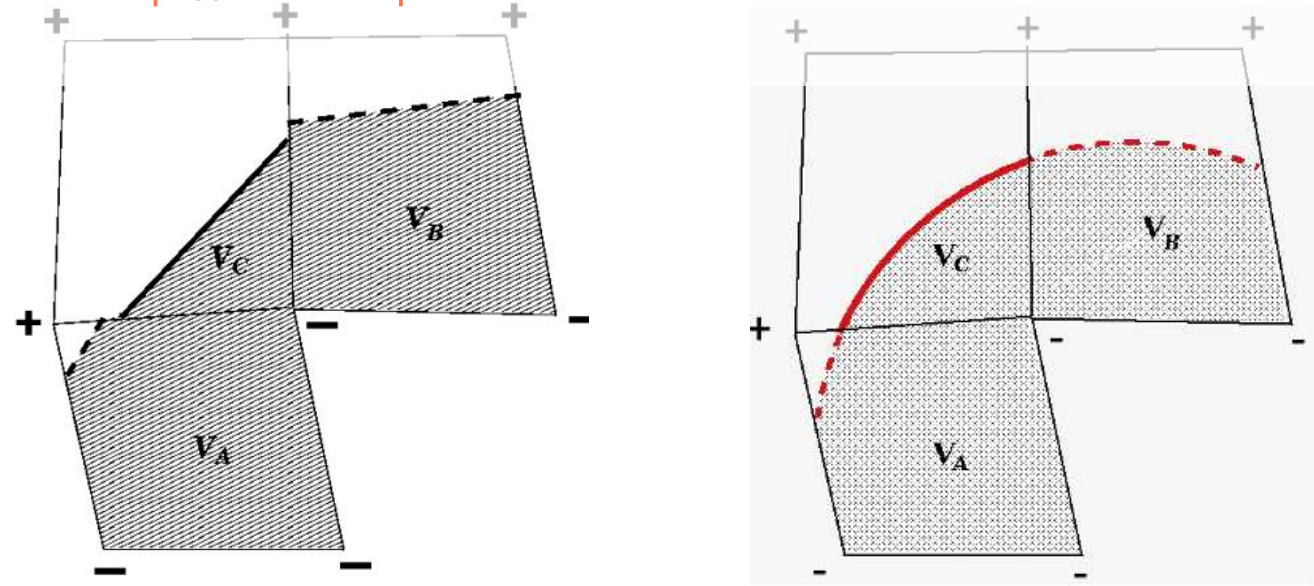

Figure 2: Regular mixed zone $\mathrm{C}$ and its neighbors $\mathrm{A}$ and $\mathrm{B}$ determined by node colors ("+" is above interface, “_“" is below). $\mathrm{V}_{\mathrm{A}}, \mathrm{V}_{\mathrm{B}}$, and $\mathrm{V}_{\mathrm{C}}$ are the corresponding partial volumes. Left: linear facets; right: circular facets. 


\subsection{Degrees of freedom and the order of a facet}

With a regular mixed zone $z C$, as described above, three local neighbors $z_{A}, z_{B}, z_{C}$ are identified and ordered with a "walk" that keeps interior (the "full" side) on the right. Thus, three partial volumes $v_{A}, v_{B}, v_{C}$, are also provided. Because a circle has three degrees of freedom, one is able to fit a circle to the local partial volumes available exactly to obtain an arc. In the case the partial volumes are consistent with a straight-line local geometry, a linear facet would be obtained, which is a degenerated case of a circle.

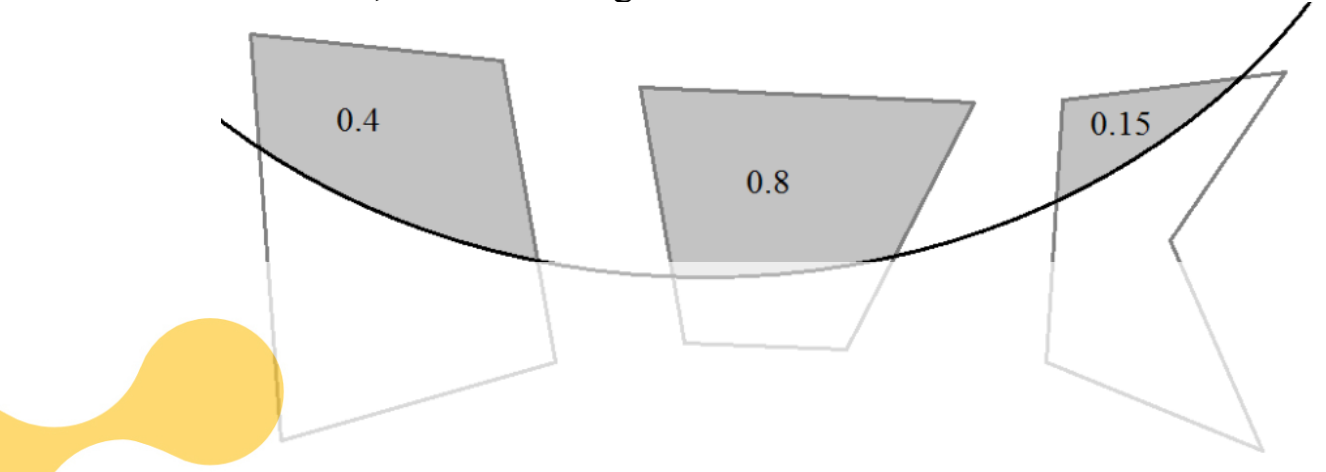

Figure 3: Three partial volume fractions uniquely determine a circle with three degrees of freedom.

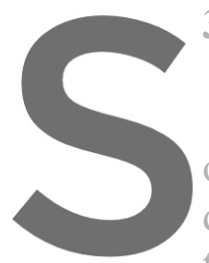

\subsection{Partial volume matching and a near quadratic con

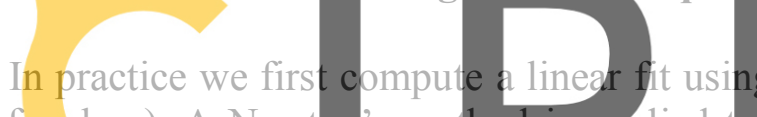 \\ of freedom). A Newton's method is applied \\ derivative of partial vol \\ to also be matched by this linear solution, no f
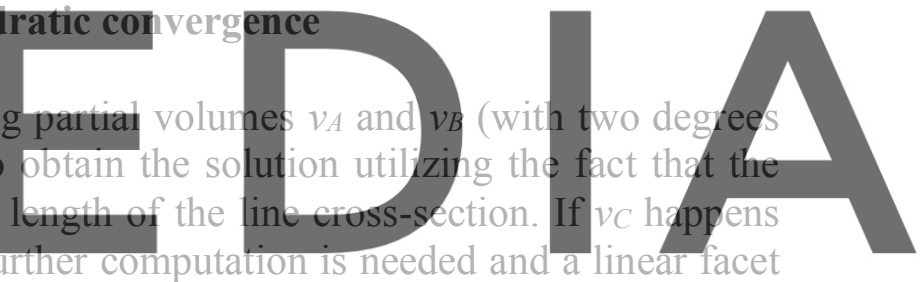

in $z C$ is obtained. Otherwise, the above linear fit is used to set the initial guess of a circular fit.

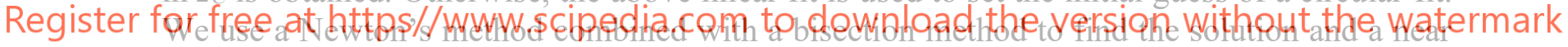

quadratic convergence is consistently observed.

\subsection{Treatment of isolated non-regular mixed zones}

For a given regular mixed zone, either a linear or a circular facet can be obtained with matching neighbor partial zone volumes. This means the solution of interface facets in most mixed zones can be obtained locally. Importantly, such a solution is final. Non-regular mixed zones that contain uncolored nodes are assumed isolated in an input mesh. The orientation of a facet in a non-regular mixed zone can be predicted with the solution of the nearest regular facet and corrected by volume matching.

\subsection{Treatment of a double-crossed edge}

It is possible that an edge of a mixed zone is crossed twice by the interface and the node colors would not help the reconstruction. In this case we merge the two mixed zones involved and treat it as a single zone without changing the method. This could mean that the partial volumes in these two zones are not matched individually. In such a situation we consider it a resolution issue and tolerant a possible loss of local accuracy. 


\subsection{Reconstructing a corner}

Corners are also assumed isolated with a sufficiently fine given mesh resolution. A corner is identified when a circle fitting fails to give a solution consistent with local volume fractions or the solution violates the assumption of moderate curvature of a facet. A corner is properly determined by adjusting the tip and letting each of its two sides pass a known solution point on a closest regular facet. The volume matching is done with a local optimization.

\subsection{A seamless interface reconstruction in 2D}

The procedure described previously would exactly reconstruct a planar polygon consist of linear or circular edges if the input volume fractions are consistent with such a polygon. In the case that an interface is not circular however smooth, the proposed reconstruction method would give a $3^{\text {rd }}$ order spatial accuracy. This means the gaps between the curved facets would be very small because of a moderate curvature. One is able to adjust the reconstructed interface facets by setting its end points at the middle of gaps and rematching the partial zone volume (fig. 4). The case of a gap is not on the same edge of a zone is rare and can be treated by setting a facet end at the common node of two edges. This operation shall provide a seamless interface which stays $3^{\text {rd }}$ order accurate in space.
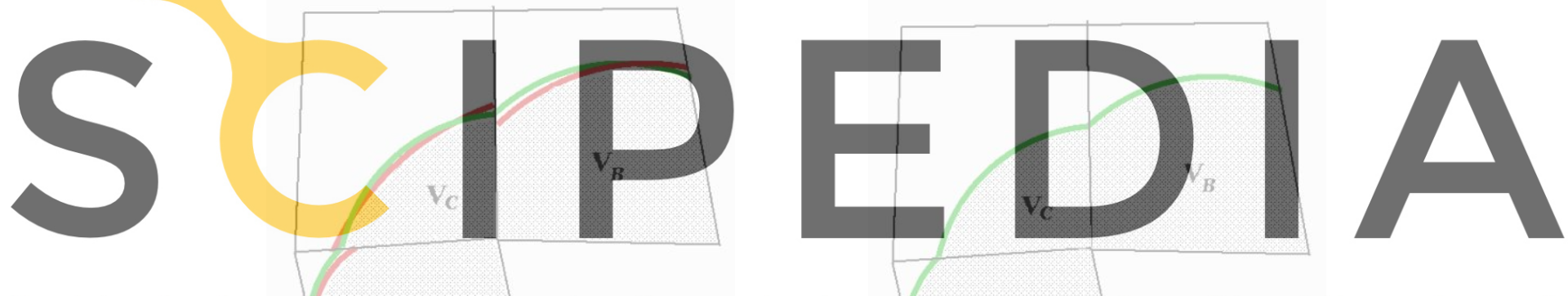

Register for free at https//www.scipedia.com to download the version without the watermark
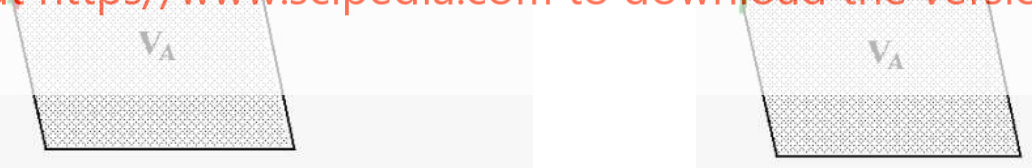

Figure 4: The red arcs are reconstructed from volume matching (left). One takes the midpoints of gaps as the new end points and matches a given volume with an arc (with three degrees of freedom) to close the gaps (right). The slope changes in the plots are much exaggerated. In all our tests the gaps between red facets are not visible.

\subsection{Boundary treatment}

In the case that a mixed zone is on mesh boundary, but no boundary condition is specified, a circular fit can still be performed to three neighbors with the said zone on a side. With a provided boundary condition, an arc can be fitted with two neighbor partial volumes. 


\section{EQUIVALENCE OF RECONSTRUCTION AND DIRECT TRACKING}

A circular facet (an arc) can be defined by three control points (say, two end points and a middle point). Each of these points is associated with a pair of logical parameters that map a quadrilateral zone to a logical square. In the Lagrange phase of an ALE advection, a circular facet is carried over with the logical parameters of the control points. The orientation is set by the order of these points. A seamless interface stays seamless after a Lagrange step.

The volume fluxes distributed into relaxed mesh are computed by finding intersections between advected and relaxed mesh bounded by the interface carried over for remapping. Then, the new partial volumes in the relaxed mesh are computed and employed for interface reconstruction at the next time step.

We point out that a seamless interface directly carried through the Lagrange motion is by nature a solution of interface reconstruction on relaxed mesh, because all the partial volumes in relaxed zones are naturally matched. In this sense, interface tracking, and its VOF (volume of fluids) reconstruction are equivalent. The only difference is the interface carried over may not be the converged solution of the interface reconstruction method of choice. Nevertheless, the information carried over can be taken as granted and used as an initial guess to accelerate the convergence of our reconstruction scheme. Furthermore, complex interface geometries difficult to reconstructe from only volume fractions can be easily tracked. Thus, combining a

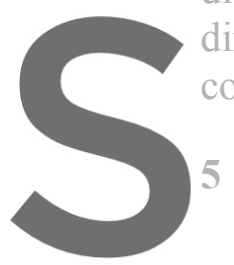
direct interface tracking (free prior info) and a vo
consistent set of facets) would provide a rather r
5 NUMERICAL RECONSTRUCTING OF

In all our numericat tests exact volume fraction

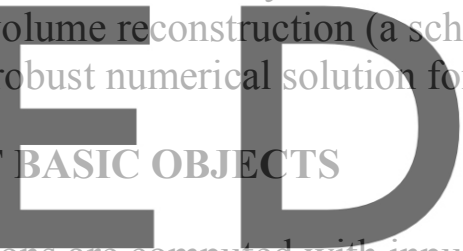

used as input for the proposed interface reconstruction scheme.

Register for free at https//www.scipedia.com to download the version without the watermark

\subsection{Reconstruct a triangle}
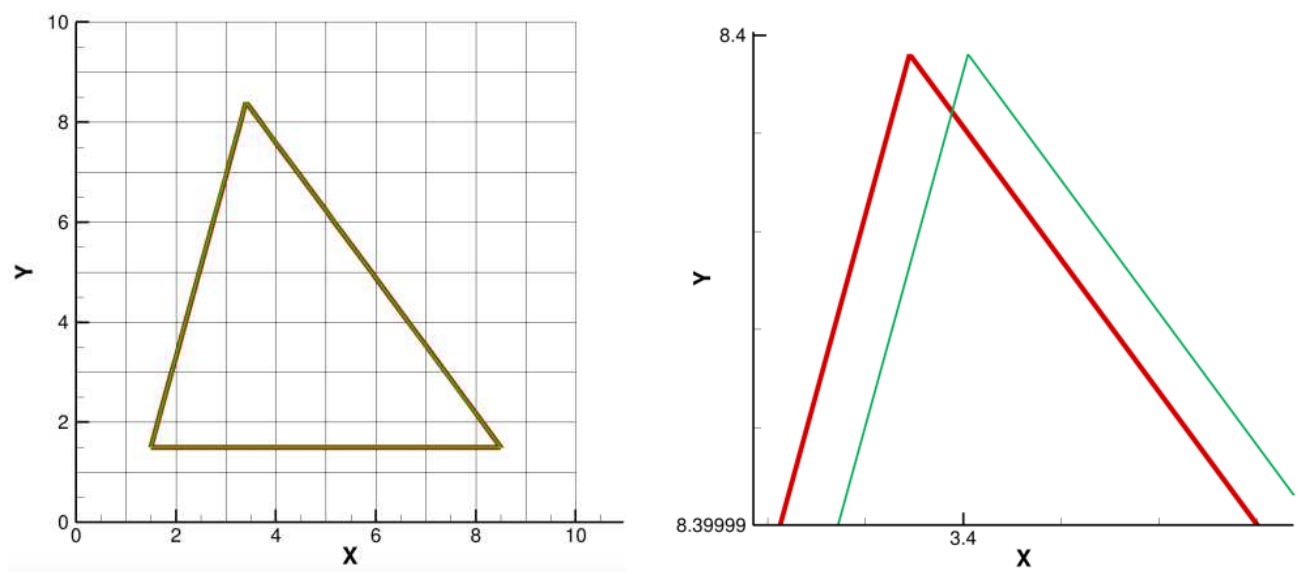

Figure 5: A green triangle defined by points $(1.5,1.5),(8.5,1.5)$ and $(3.4,8.4)$ is used to compute volume fractions in a Cartesian mesh. The red is reconstructed with the proposed method with a tolerance of $10^{-6}$. The area of right plot is $10^{-12}$ of the left one. In theory the solution would be exact with an infinitely small tolerance. 


\subsection{Reconstruct a circle}

We implemented the proposed method first using a parabola facet defined on a local Cartesian coordinate by setting the Youngs facet as the horizontal axis with its midpoint as the origin, and the vertical axis in the facet normal direction. The reconstruction of circle shown below is obtained with volume fittings using parabola facets. We later decide to employ circular facets because no local coordinate system is needed.
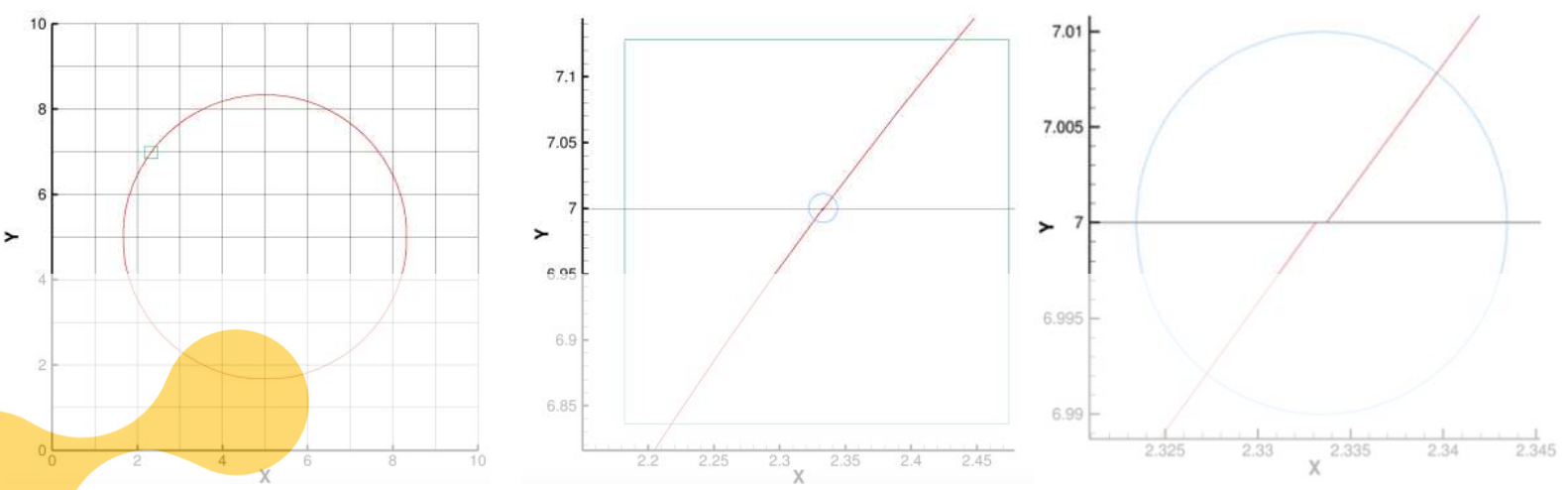

Figure 6: A circle centered at $(0,0)$ with a radius $10 / 3$ is reconstructed on a unit Cartesian mesh (the red circle on left). The middle plot is the small squarish region on the left zoomed in. The right plot is the circular region in the middle plot zoomed in. A tolerance of $10^{-6}$ is used for a parabola (not a circle) fitting with neighbor volumes.

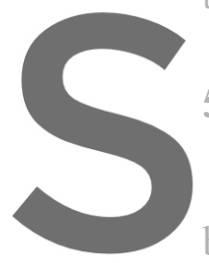

5.3 Reconstruct $2 \mathrm{D}$ dbjects on perturbed mashes
The proposed interface reconstruction method does not require below. It should work with an a mesh structure with the proposed method.

Register for free at https//www.scipedia.com to download the version without the watermark
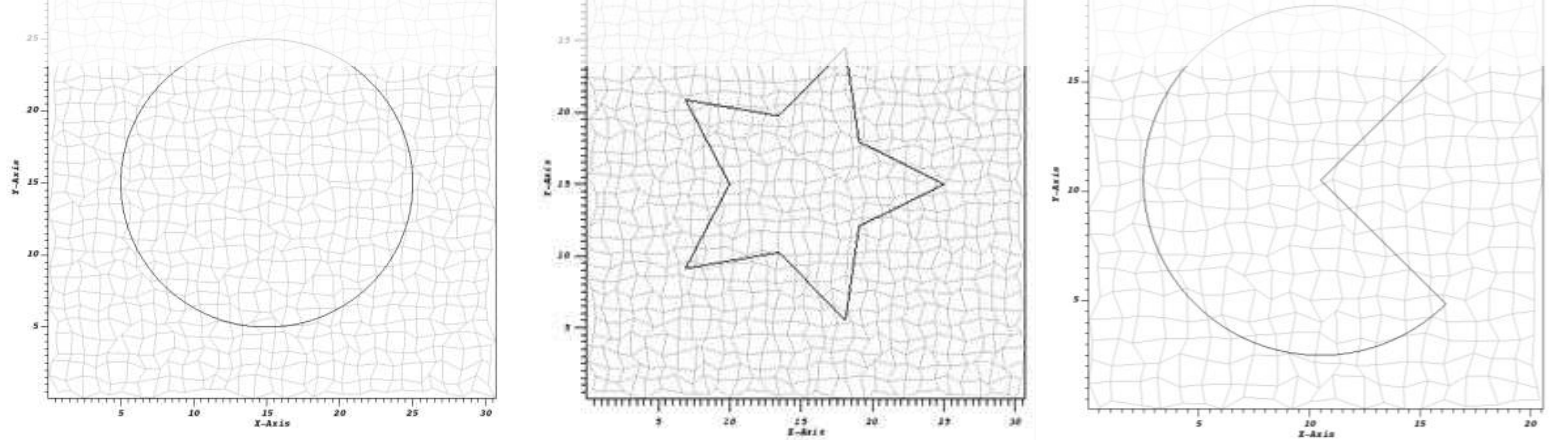

Figure 7: Interface reconstructions with the new method on perturbed Cartesian meshes (with a tolerance $10^{-11}$ ).

\section{$5.4 \mathrm{~A} 3^{\text {rd }}$ order accuracy of the proposed method}

The proposed new interface reconstruction method consistently shows a $3^{\text {rd }}$ order spatial accuracy in all the tests we have tried. This is expected because we employed a curved facet geometry with three degrees of freedom. We demonstrate this by reconstructing an ellipsoid. 

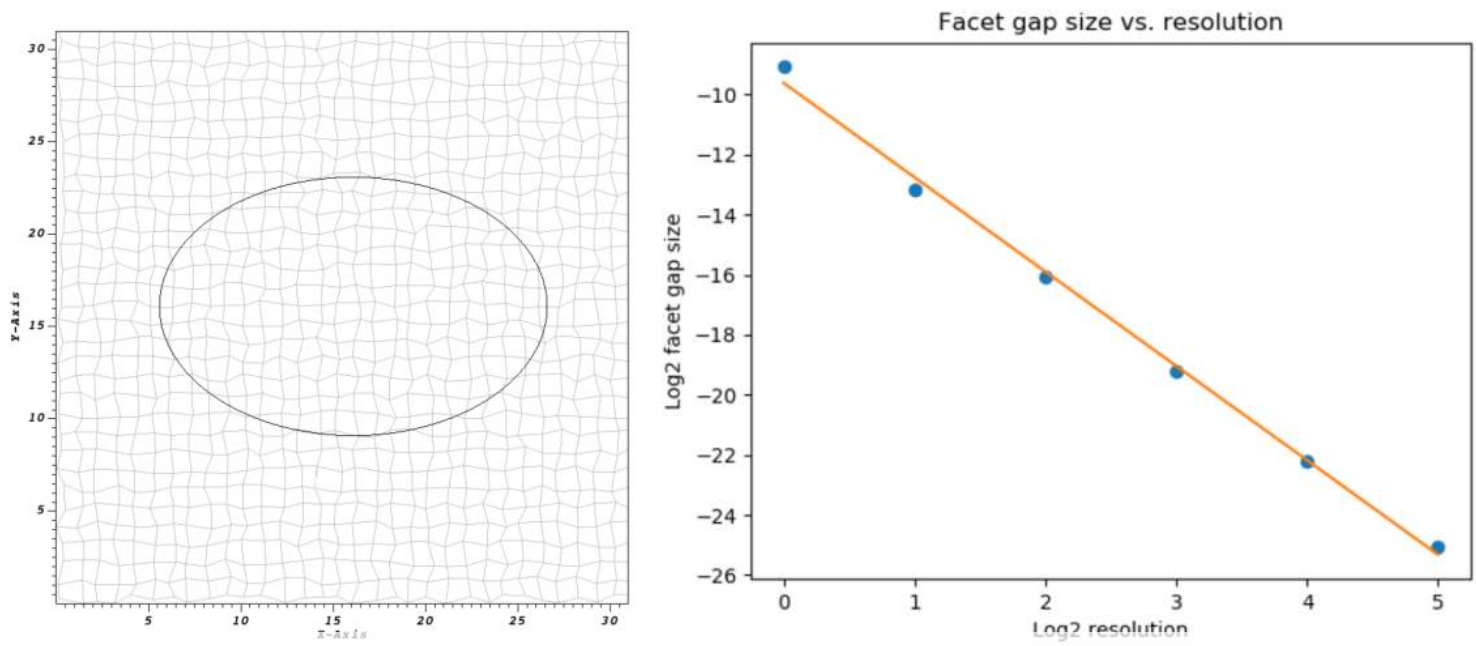

Figure 8: An ellipsoid is reconstructed on a perturbed Cartesian mesh (left). The slope of $\mathrm{L}_{2}$ gap sizes vs. resolution is plotted in a logarithm scale (the base is 2) on the right side and a slope of -3 is clearly shown.

\section{6}

\section{NUMERICAL VOLUME ADVECTION TESTS}

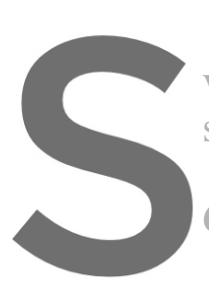

\footnotetext{
We have tested the
}

volume advection probl

set back to unit Cartesia

6.1 With a single material
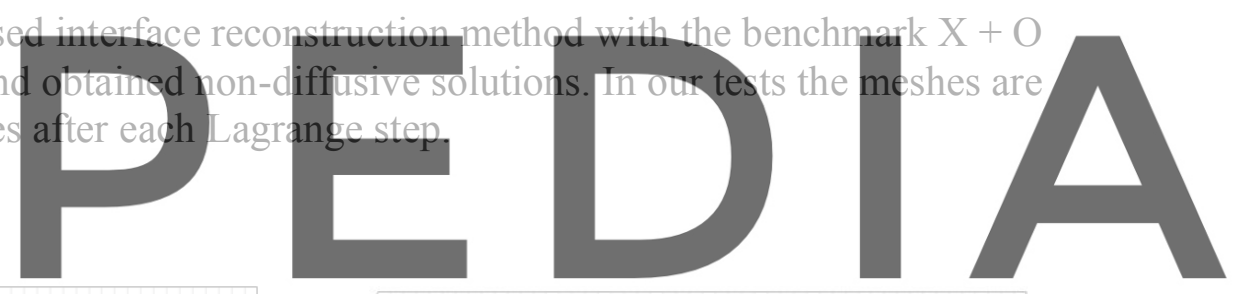

Register for free at https//www.scipedia.com to download the version without the watermark
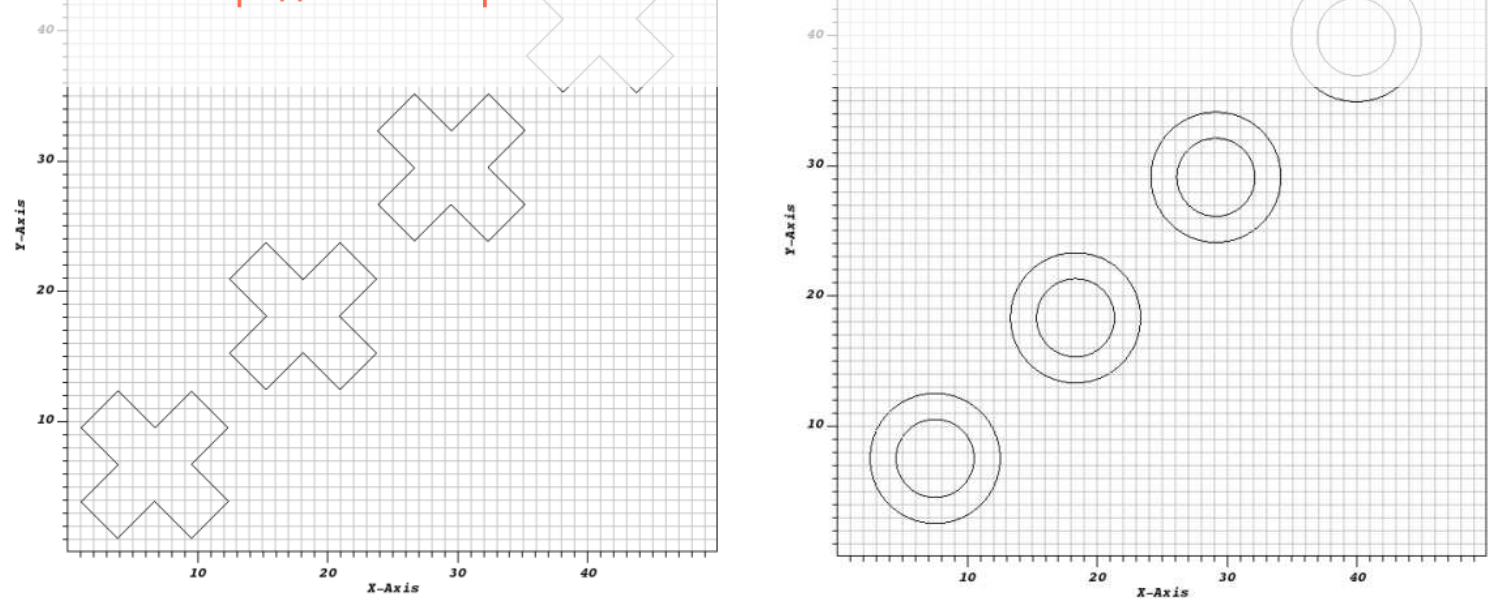

Figure 8: Diagonal translation of 2D objects. Object positions are shown at four different moments. Velocity is set to constant $(1,1)$ and time step is set to 0.1 . No prior information is used to help the interface reconstruction. 


\subsection{With multiple materials}

There are three materials (blue, green, void) in the advection test below. We reconstruct the interface with a single material vs. the rest fashion then assemble the results. In most cases a mixed zone is regular and contains a single piece of interface. Then, a single reconstruction is sufficient. The material ordering info can be carried over time steps, but we did not utilize it.

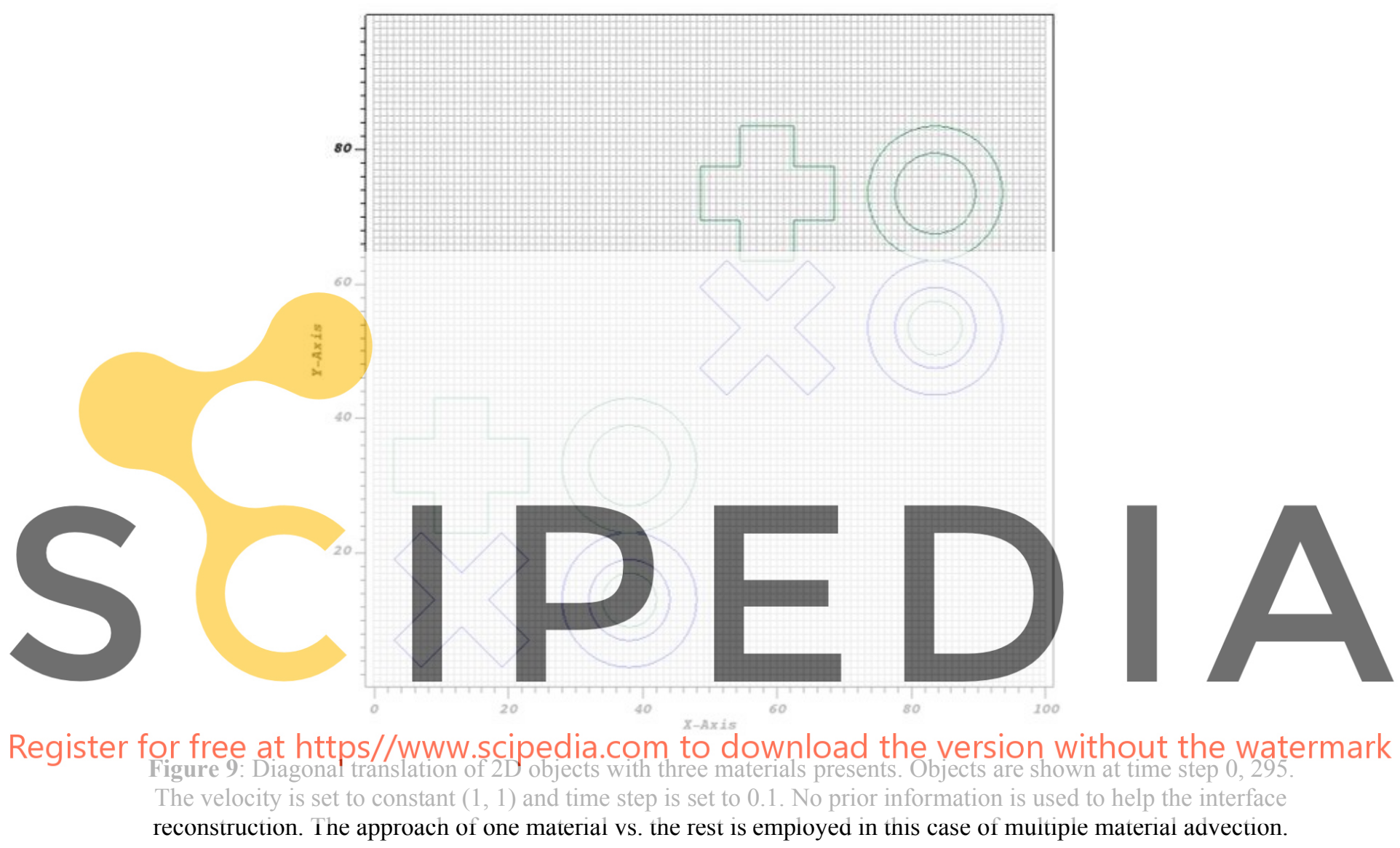

We have obtained non-diffusive solutions of other benchmark advection tests too. We are also able to treat double curved facets inside a single mixed zone with the help of prior interface info carried over each time step. We shall have this developed advection scheme explained in detail in a different article.

\section{CONCLUSIONS}

- We propose to construct curved (circular) interface facets instead of linear ones in order to remove some historic issues of Youngs method. We obtain the orientation of a regular mixed zone with coloring mesh nodes by pure zone material IDs. Most of mixed zones are regular (i.e., contains a single moderately curved or straight interface piece) with a good input mesh resolution. We utilize the fact that a circle has three degrees of freedom and use the three partial volumes of the immediate neighbors of a 
regular mixed zone (including itself) to fit a circular facet (an arc). We match local volume fractions with a combination of Newton's method and a bisection method to compute the solution in smooth portion and observe a near quadratic convergence consistently. A facet in a non-regular zone is predicted with the solution in its closest regular neighbors and corrected by local volume matching. Isolated corners can also be accurately reconstructed with local optimization. A seamless interface can also be constructed in two dimensions with a third order spatial accuracy.

- We demonstrated the effectiveness and accuracy of the new interface reconstruction method by comparing the solution of reconstruction with exact solutions using basic geometrical shapes. We applied the new interface reconstruction method in some benchmark volume advection problems and achieved a non-diffusive advection.

- We point out that reconstructing a seamless interface and carrying it over a Lagrange step are equivalent because a direct tracking by nature allows exact matching of all the partial volumes in relaxed mesh. As a result, a complex interface geometry can be advected accurately utilizing prior geometrical information granted in an ALE simulation.

We believe the proposed interface reconstruction scheme can be extended into three dimensions if an accurate method to compute 3D partial volumes bounded by conic surfaces is available.

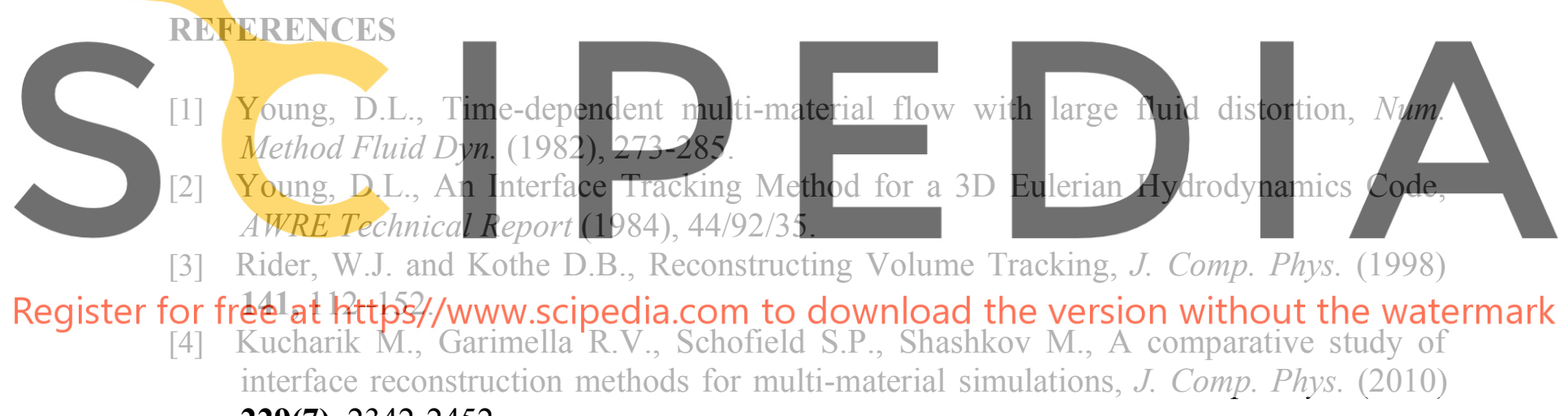

229(7), 2342-2452.

[5] Yao J., A localized accurate VOF interface reconstruction method with curvature and corner definitions in two dimensions, Technical Report (2020), LLNL-TR-801883.

[6] Maity, R.K., Sundararajan, T., Velusamy, K., An accurate interface reconstruction method using piecewise circular arcs, Num. Methods in Fluids (2021), 1, 93-126.

This work was performed under the auspices of the U.S. Department of Energy by Lawrence Livermore National Laboratory under Contract DE-AC52-07NA27344. 